\title{
Análisis crítico del discurso: narrativas sobre violencia de género en un estudiante universitario de una institución de educación superior del sur de Bogotá (Colombia)
}

\author{
Elizabeth Moreno-Gómez y John J. García-Díaz \\ Facultad de Educación, Corporación Universitaria Minuto de Dios - UNIMINUTO, calle 1, número 9 - 50, Bogotá D.C., \\ Colombia. (Correo-e: eliza.moren@gmail.com; seilla4@gmail.com)
}

Recibido Sep. 22, 2020; Aceptado Nov. 18, 2020; Versión final Feb. 5, 2021, Publicado Jun. 2021

\begin{abstract}
Resumen
Este artículo analiza la violencia de género presente en el discurso de un estudiante de educación superior en un contexto universitario al sur de Bogotá (Colombia). La metodología consiste en hacer un análisis crítico del discurso y de contenido para identificar los diferentes tipos de violencia de género y las relaciones de poder en la transcripción de una entrevista. Los resultados indican que el discurso es $60 \%$ de víctima, $20 \%$ de victimario y $20 \%$ de testigo directo de los hechos. Su estructura de exposición es expresiva en bullying, representativa en acoso y abuso sexual, y comisivo y expresivo en rechazo y exclusión social. Se concluye que las relaciones de poder son desiguales, el principal victimario es el hombre, las víctimas son mujeres y la diversidad sexual, y que los tipos de violencia son psíquica, social y sexual. Finalmente, el presente estudio pretende contribuir a la discusión sobre la equidad de género en la educación superior.
\end{abstract}

Palabras clave: violencia; género; discurso; análisis crítico; educación superior; relaciones; poder

\section{Critical analysis of speech: narratives about gender violence by a university student at a higher education institution from southern Bogotá (Colombia)}

\begin{abstract}
The present study examines gender violence in the speech by a higher education student from southern Bogotá (Colombia). The study methodology includes a critical analysis of speech and content to identify different types of gender violence and power relations in an interview transcript. The results show that the speech is $60 \%$ victim, $20 \%$ victimizer, and $20 \%$ direct facts witness. The speech's exposure structure is expressive in bullying, representative in sexual harassment and abuse, and commissive and expressive in rejection and social exclusion. It is concluded that power relations are unequal, the main victimizer is a man, the victims are women and sexually diverse, and the types of violence are psychological, social, and sexual. Finally, the present study aims to contribute to the discussion on gender equality in higher education.
\end{abstract}

Keywords: gender violence; speech; critical discourse analysis; higher education; power relationships 


\section{INTRODUCCIÓN}

Ilustrar la implementación de la metodología del análisis crítico del discurso (ACD), en un estudio sobre violencia de género en el contexto educativo, implica reconocer su utilidad en el análisis y la interpretación de las relaciones de poder y su aporte en investigaciones acerca del sentido y significado de una realidad social y cultural. Por ende, de manera simultánea se describen los pasos de la puesta en escena de la técnica y su uso en esta pesquisa. La violencia de género es un fenómeno sociocultural asociado a diferentes hechos de dominación y sometimiento que a través de la agresión refuerza el "dominio masculino sobre el cuerpo de las mujeres" (Ballester, 2012). Esta involucra comportamientos y actitudes sustentadas en las relaciones de poder que hieren, amenazan y debilitan a las personas por causa de su género o sexualidad (Lewis y Anitha, 2019). El concepto se caracteriza por hacer referencia en su interior a dos roles: el agresor (sujeto dominantemanipulador) y la víctima (sujeto maltratado-manipulado).

En Colombia, por su parte, la violencia de género ha aumentado en los últimos años; por ejemplo, para el año 2015 se reportaron 36.206 víctimas de violencia física, 17.376 violencia sexual y 4.807 violencia psicológica y en el 2018, 52.548 las víctimas de violencia física, 29.804 violencia sexual y 8.700 violencia psicológica (SIVIGE, 2018). Esta violencia se puede dar en diversos contextos, entre ellos el universitario, donde se observan dinámicas de poder desigual, estas son, de acuerdo con San Segundo y Codina-Canet (2019), "relaciones de abuso, de acoso, de dominación, estas últimas son las relaciones que se establecen en la violencia" (p. 29). Dichas dinámicas visibilizan en el rol de víctima con mayor afectación a las mujeres y en el papel del agresor motivado por el odio, al hombre (Lewis y Anitha, 2019).

Existen investigaciones sobre la violencia de género en la educación superior, como, por ejemplo, la de Álvarez-Aguilar et al. (2019) quienes realizaron un estudio sobre los factores sociales y personales en mujeres estudiantes de ingeniería, programa que presenta el menor porcentaje de matrícula de mujeres; se encontró que los factores sociales determinantes en correspondencia con la admisión, permanencia y proyección al futuro se encuentran relacionados con machismo, discriminación, acoso, estereotipos y prejuicios sociales, en cuanto a las consecuencias interpersonales generadas se encuentra efectos negativos de inseguridad, miedo y autoestima, que resultan ser consecuencias de los factores sociales. En ese sentido, la pesquisa de Vandana (2020) acerca de género (mujeres) y casta (superiores o clases dominantes), exploró las experiencias de acoso sexual, en las interacciones sociales, vividas por mujeres Dalit (personas que se consideran inferiores en el sistema de castas) en el campus universitario, frente a lo cual se resaltan las maneras en que las niñas Dalit artículan e interpretan el acoso a la vez que procuran educación superior.

De igual manera, en un proyecto de investigación social con intervención en educación superior se encontró evidencia de pautas culturales que reproducen hechos de violencia de género que afectan las experiencias de las mujeres y las minorías sexuales en instituciones universitarias, donde el contexto respalda aquellas experiencias negativas (Lewis y Anitha, 2019). Así mismo, adicionar a dichas pautas culturales la educación familiar da como resultado un mayor impacto en las actitudes de igualdad de género, tal como lo plantean en su análisis Tekbas y Pola (2020). Otro estudio encontrado en la revisión trata sobre el análisis del bullying homofobico a estudiantes en contextos de educación superior el cual se sustenta en la construcción y búsqueda de una dialéctica entre la resistencia y la violencia (Ruiz, Evangelista y Xolocotzi, 2018).

Por otro lado, el estudio de Aguiar et al. en 2020, buscó comprender el significado de los ataques sexuales contra las mujeres desde la mirada de los estudiantes, docentes y directivos universitarios de enfermería; al respecto, se encontró que estos son un fenómeno frecuente, complejo y multidimensional, las principales agresiones en orden de mayor a menor incidencia son la violencia moral, psicológica, sexual, física y patrimonial. En la investigación de Ramos-Jiménez et al. (2017) sobre la interpretación del uso del bullying por género y el nivel de educación en un sector vulnerable en México, se halló que las principales manifestaciones de este tipo de violencia fueron la burla, exclusión social, culpa, amenazas, castigos y golpes, los cuales disminuyen con el grado académico y la edad; estas acciones son ligeramente más recurrente en hombres y afectan las relaciones saludables entre los estudiantes, puesto que generan entornos de inseguridad y agresividad.

Además, investigaciones como las de García-Díaz et al. (2020) y Kelmendi y Baumgartner (2020) en universidades sobre violencia entre parejas, encontraron que, por un lado, en España es muy alta la tolerancia a las actitudes sexistas y violencia en pareja y, por otro lado, que en Kosovo, la aprobación de la violencia intercede en medio de la socialización de la violencia y la perpetración y victimización para ambos sexos, aparte, se observan diferencias de género estadísticamente significativas en socialización y aprobación de la violencia. En suma, los dos trabajos proponen discutir estos hallazgos en el contexto formativo de la educación superior. Así, por ejemplo, lo expresan Osuna-Rodríguez et al. (2020) al cosiderar que la educación es la estrategia más efectiva en la erradicación de la discriminación y violencia contra la mujer, y Maito et al. (2019) al plantear un instrumento institucional para enfrentar la violencia contra las mujeres. 
Por último, las investigaciones sobre violencia de género y diversidad sexual son importantes, puesto que en ellas se evalúa el impacto social durante el procesos de formación y las experiencias de violencia de género en el entorno formativo (Devís-Devís et al. 2018). En la revisión realizada, se encontraron pocas investigaciones sobre el tema en instituciones de educación superior. En consecuencia, el presente artículo tiene como propósito describir las etapas del análisis crítico del discurso a través del análisis de una narrativa sobre violencia de género de un estudiante universitario de una institución de educación superior del sur de Bogotá.

\section{METODOLOGÍA}

El objeto de estudio se seleccionó de un conjunto de entrevistas realizadas a estudiantes de cinco licenciaturas ofertadas en instituciones de educación superior privadas del sur de Bogotá D.C., en el primer semestre del año 2019. El estudio principal se enfoca sobre el análisis de una narrativa de un estudiante en educación superior, mayor de edad, que en ese momento era parte del programa de Licenciatura en Artes Escénicas.

\section{Análisis de los datos}

El análisis de contenido, es una técnica sistemática de análisis (Fernández, 2002; Ahmad y Shah, 2019) del lenguaje, hablado o escrito, con el propósito de comprender el sentido y significado de la realidad social y cultural a través de la información recolectada durante el estudio. Por todo lo expresado, el lenguaje ocupa en este proceso el papel principal, es decir, por un lado, a partir de él, se construye el sentido y significado de las ideas plasmadas en los escritos (discursos) y, por otro lado, al ser considerado como práctica social permite establecer las relaciones de poder que se manifiestan en la intersección del contexto educativo y el género, en concreto, violencia de género.

\section{Análisis crítico del discurso}

Este estudio realizó un ACD de acuerdo con los postulados de Woka (2003) y Van Dijk (2000), esto es, el análisis crítico de "las relaciones de dominación, discriminación, poder y control" (Woka, 2003, p. 19) en los discursos (Van Dijk, 2000; Dalton, 2019) de un estudiante de educación superior en el contexto universitario masculino dominante. Así pues, dicho método permitió definir para su implementación el siguiente proceso:

\section{Modelo para el análisis}

Para la interpretación de los datos, esta investigación usó las etapas propuestas por Fernández (2002), con modificaciones en la implementación. Dicha propuesta permitió desarrollar la técnica de análisis en seis etapas, a saber: 1) la población se conformó por 31 entrevistas a estudiantes de licenciatura en artes escénicas, educación física, educación básica con énfasis en ciencias sociales, ciencias naturales y matemáticas de tres instituciones de educación superior al sur de Bogotá D.C.; 2) la muestra se constituyó por una entrevista de un estudiante de la licenciatura en artes escénicas mayor de edad; 3) la unidad de análisis se seleccionó de la conceptualización del estudio, o sea, la violencia de género; 4) la unidad de contexto se compuso por bullying, acoso o abuso sexual, discriminación, rechazo y exclusión social; 5) la categoría se integró por víctima y victimario; y 6) el análisis se desarrolló desde dos enfoques, esto es, cuantitativo y cualitativo. El primero se ejecutó con el fin de codificar y cuantificar los elementos de la unidad de contexto, vale decir, el número de registros o apariciones en el discurso. El segundo se realizó con el propósito de hallar la asociación descrita por Woka (2003) entre el análisis de contenido y el análisis de la estructura del discurso. Por ello, el ACD se dividió en dos. En la figura 1 se presenta el proceso metodológico que se llevó a cabo para el ACD.

En primer lugar, el análisis de contenido. Este proceso tuvo en cuenta el tipo de discurso (víctima, victimario o testigo directo), los acontecimientos y el contexto discursivo bajo el cual el entrevistado expuso su comunicación. Para el análisis de la información, se implementaron el proceso cíclico de selección de unidades de estudio, la ubicación de preguntas de la entrevista, el discurso textual del sujeto, la referencia de la teoría y la correlación e interpretación. Dicho proceso se realizó junto con la creación de la matriz de filtro para ubicar la categoría, la relación sujeto-categoría, el tipo de sentimientos (positivos, negativos o neutros), la forma de agresión y el contexto universitario, este análisis se consignó en una matriz de análisis de datos. Finalmente, se cuantificó el número de sujetos identificados en el discurso y la frecuencia de aparición por categoría.

En segundo lugar, el análisis de la estructura. El mencionado análisis situó para cada una de las unidades de contexto la pregunta y discurso textual del sujeto, luego, esa información fue separada en dos partes, a saber: fragmentos discursivos y enmarañamiento del hilo discurso. En este punto, es necesario precisar que el primero corresponde a la textualidad de la respuesta a la pregunta y el segundo, responde a los elementos 
que acompañan la respuesta de la pregunta, pero que no tiene una relación directa con la pregunta y son mencionados en el discurso del entrevistado. De esta manera, se considera que el ACD de un estudio de caso podría emplearse como estrategia de detección de violencia de género en educación superior, lo que permitiría eliminar paulatinamente hechos que han sido aprendidos social y culturalmente.

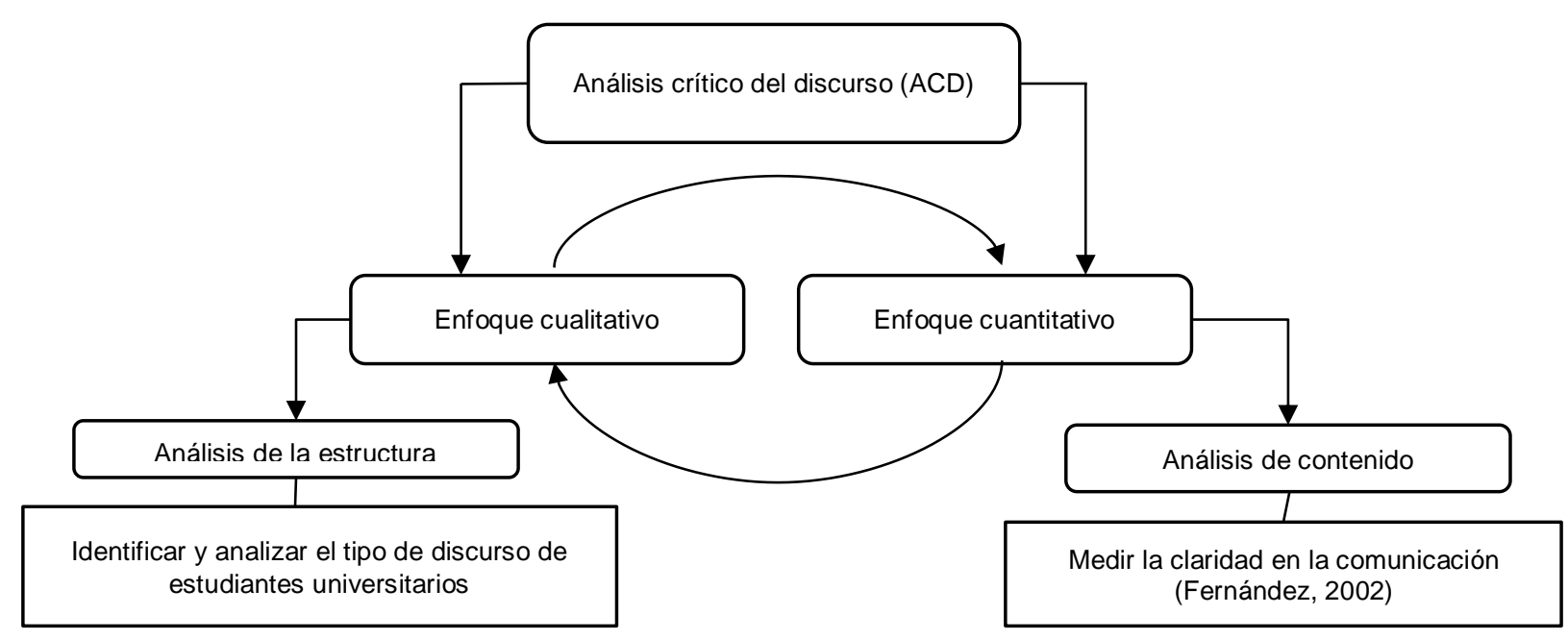

Fig 1: Componentes del diseño metodológico en el ACD.

\section{RESULTADOS}

A continuación, se presentan los resultados del ACD a través de los fragmentos discursivos en relación con las unidades de contexto, sobre violencia de género. En la tabla 1 se presenta el consolidado del ACD, desde el enfoque cuantitativo. Así mismo, esta posee en la columna sujetos un asterisco que indica la frecuencia en el discurso. El origen de los datos en las tablas 2,3 y 4, son de la transcripción de la entrevista realizada al sujeto de la investigación.

Tabla 1: Resultados cuantitativos del análisis crítico del discurso.

\begin{tabular}{|c|c|c|c|c|c|}
\hline Discurso & $\begin{array}{l}\text { Unidades de } \\
\text { contexto }\end{array}$ & Sujetos* & Sentimientos & Tipo de agresión & Contextos universitarios \\
\hline \multirow[t]{3}{*}{ Víctima } & Bullying & $\begin{array}{l}\text { Maestros (1) } \\
\text { Hombres (4) Profesores (10) } \\
\text { Amigos (2) } \\
\text { Machos (1) } \\
\text { Mujer (2) } \\
\text { Homosexuales (7) Lesbianas (1) } \\
\text { Heterosexual (1) }\end{array}$ & Negativos & $\begin{array}{l}\text { Imitación, burla, sabotaje, } \\
\text { morbo, chistes y bromas }\end{array}$ & $\begin{array}{l}\text { Actividades académicas } \\
\text { del programa. Espacios de } \\
\text { socialización (cafeterías y } \\
\text { pasillos) }\end{array}$ \\
\hline & $\begin{array}{l}\text { Acoso, abuso } \\
\text { sexual }\end{array}$ & $\begin{array}{l}\text { Maestro (1) } \\
\text { Hombre (5) } \\
\text { Mujeres (2) } \\
\text { Amigos (1) } \\
\text { Homosexual (1) }\end{array}$ & Negativos & $\begin{array}{l}\text { Presión psicológica sobre } \\
\text { temas académicos }\end{array}$ & $\begin{array}{l}\text { Actividades académicas } \\
\text { del programa académico }\end{array}$ \\
\hline & $\begin{array}{l}\text { Discriminación, } \\
\text { Rechazo, } \\
\text { exclusión } \\
\text { social }\end{array}$ & $\begin{array}{l}\text { Maestro (3) } \\
\text { Profesores (1) Hombre (22) } \\
\text { Macho (3) } \\
\text { Mujer (6) } \\
\text { Homosexuales (3) } \\
\text { Heterosexual (1) }\end{array}$ & Negativos & $\begin{array}{l}\text { Descalificación y } \\
\text { estereotipo }\end{array}$ & $\begin{array}{l}\text { Actividades académicas } \\
\text { del programa académico }\end{array}$ \\
\hline Victimario & Bullying & $\begin{array}{l}\text { Profesores (1) } \\
\text { Profesoras (1) homosexuales (1) }\end{array}$ & Neutros & $\begin{array}{l}\text { Imitación, chistes y bromas } \\
\text { de mal gusto }\end{array}$ & $\begin{array}{l}\text { Actividades académicas } \\
\text { del programa académico }\end{array}$ \\
\hline Testigo directo & $\begin{array}{l}\text { Acoso, abuso } \\
\text { sexual }\end{array}$ & $\begin{array}{l}\text { Docentes (1) } \\
\text { Maestro (2) } \\
\text { Compañeros (2) Hombres (1) } \\
\text { Mujer (1) }\end{array}$ & $\begin{array}{l}\text { Afecto, } \\
\text { afinidad }\end{array}$ & Sabotaje & $\begin{array}{l}\text { Espacios de formación del } \\
\text { programa académico }\end{array}$ \\
\hline
\end{tabular}


Tabla 2: Violencia de género: fragmento discursivo bullying

\begin{tabular}{|c|c|}
\hline Pregunta & Fragmento discursivo \\
\hline $\begin{array}{l}\text { Pregunta 1: ¿Conoces en qué escenarios de la } \\
\text { universidad se producen burlas, insultos, rechazo, } \\
\text { crítica, imitación, y sabotaje? ¿Por qué? ¿Cómo se } \\
\text { produce la situación? }\end{array}$ & $\begin{array}{l}\text { "Siento que no es como tal en un lugar específico, } \\
\text { porque siento que todos los lugares pueden ser esos } \\
\text { espacios y, por ejemplo, pasa acá en la cafetería..." } \\
\text { "entre personas que son homosexuales, que son } \\
\text { bisexuales, no sé, lo que te decía, entonces el chiste y la } \\
\text { chanza y, entonces, "Ahí llegó mi amiga la perra” } \\
\text { entonces "Ay si" y bueno entonces yo me rio y ya..." } \\
\text { "...pero si, definitivamente es una falta de respeto, si es } \\
\text { una burla "Ahí que llegó...” y el chiste de que llegó y } \\
\text { camina así, entonces, el chiste y la misma persona a la } \\
\text { que le hicieron el chiste se ríe, porque ese es el chiste..." } \\
\text { "...si hay un chiste es de cualquier persona y si hay un } \\
\text { chiste es de cualquier cosa, porque no está mal que nos } \\
\text { riamos y no está mal un chiste, si es por la forma de ser } \\
\text { de de una persona eso ya es una vulneración también..." }\end{array}$ \\
\hline $\begin{array}{l}\text { Pregunta 2: ¿Has realizado comentarios, críticas, } \\
\text { burlas o bromas en contra de alguien por su género en } \\
\text { el contexto universitario? ¿Has sido testigo de alguno } \\
\text { de estos hechos? ¿A quién fue dirigida? ¿Cómo se } \\
\text { presentó dicha situación? }\end{array}$ & $\begin{array}{l}\text { “...uno busca chistes y bromas, pero uno las encuentra } \\
\text { más fácil en esas personas...” } \\
\text { “...yo tambien arremedo a las profesoras, a los } \\
\text { profesores, pero cuando hay una persona que es } \\
\text { homosexual pues obviamente se nota mucho...” } \\
\text { "...cuando es gay y, entonces, "El profe que es así y el } \\
\text { que habla así...” } \\
\text { “...Siento que no... bueno, no es que no lo haga, pero si } \\
\text { lo hago...” } \\
\text { "...uno encuentra una manera perfecta de burlarse de } \\
\text { alguien...” } \\
\text { "...Entonces si, a veces sí lo he hecho...” }\end{array}$ \\
\hline $\begin{array}{l}\text { Pregunta 3: ¿Alguna vez has visto algún } \\
\text { compañero/administrativo/docente/otro (cuál) } \\
\text { mofándose de alguien por su condición como mujer, } \\
\text { hombre, homosexual, lesbiana, transgénero? ¿A } \\
\text { quién? ¿Cómo se dio la situación? }\end{array}$ & $\begin{array}{l}\text { “...Si, y fue delicado porque yo veía profesores de la } \\
\text { universidad...” } \\
\text { "...que hagan eso, que hablen eso. Bueno, el vigilante no } \\
\text { me parece que lo haga, pero digo "bueno”, la señora del } \\
\text { aseo "bueno", pero que un docente, porque fue un } \\
\text { docente de la universidad, de este programa que es artes } \\
\text { escénicas...” } \\
\text { "...el profesor haciendo comentarios como "Ay que tal } \\
\text { esos dos besándose ahí" ¿Si? "Es que deberían, si se } \\
\text { van a besar, deberían besarse en otro lado". Entonces, } \\
\text { claro, el comentario de los profesores, una pareja } \\
\text { homosexual que hay en la universidad, entonces, los } \\
\text { profesores después comentando "Ay que este es novio } \\
\text { de este y que..." } \\
\text { "...y hay burlas también de los estudiantes...pasa mucho, } \\
\text { por ejemplo: Ay es que esa es la perra y no... es que se } \\
\text { desnudó, porque eso mejor dicho le gusta que todo el } \\
\text { mundo la mire...” } \\
\text { "...Porque un hombre heterosexual no lo hace, eso es } \\
\text { porque es gay, entonces, ahí empieza como esa cosa de } \\
\text { que hablemos de esto, digámosle esto...”" }\end{array}$ \\
\hline
\end{tabular}


Tabla 3: Violencia de género: fragmento discursivo acoso o abuso sexual.

\begin{tabular}{|c|c|}
\hline Pregunta & Fragmentos discursivos \\
\hline $\begin{array}{l}\text { Pregunta 4: ¿Podrías describir la comunicación y } \\
\text { relación con las personas que conoces en la } \\
\text { universidad (docentes, compañeros, administrativos, } \\
\text { etc.)? ¿consideras que hay algo en particular en la } \\
\text { manera en que se comunican (gestos, apodos, } \\
\text { manías, etc.)? }\end{array}$ & $\begin{array}{l}\text { “... somos muy cariñosos, o sea, acá la mayoría es } \\
\text { abrazando al otro, de pronto también por toda esta vaina } \\
\text { del arte..." } \\
\text { "...si somos como muy amigos, muy desde el campo } \\
\text { también de los artístico, entonces, siempre uno se } \\
\text { encuentra y habla de los espacios de creación, de las } \\
\text { prácticas, entonces, si hay como mucha forma de } \\
\text { afecto..." } \\
\text { "...siempre hay besos, abrazos y en la universidad } \\
\text { también, he visto a un hombre saludándose con un } \\
\text { hombre de beso en la mejilla, sin necesidad de que los } \\
\text { dos sean homosexuales..." } \\
\text { "...si en personas se da eso, como de la confianza..." }\end{array}$ \\
\hline $\begin{array}{l}\text { Pregunta 5: ¿Conoces de alguien que ha sido víctima } \\
\text { de alguna forma de acoso en la universidad? ¿De } \\
\text { qué manera se dio el acoso? ¿Verbal, social, físico o } \\
\text { través de medios tecnológicos de comunicación?? }\end{array}$ & $\begin{array}{l}\text { "Que te dijera, pues es que no sé, no sé hasta qué punto } \\
\text { pueda ser acoso porque de pronto sí, no sé si de pronto } \\
\text { en esos actos de afecto a veces uno puede ser muy..." } \\
\text { "por ejemplo los hombres, pueden ser muy reiterativos y } \\
\text { como muy encima de la chica" } \\
\text { "el comentario "Que muy linda y que no sé qué" pero } \\
\text { claro llega tambien el punto en que si claro le dice de } \\
\text { pronto, tambien se siente vulnerada. Porque es como... } \\
\text { ¿Si? Entonces eso tambien se da en la universidad. }\end{array}$ \\
\hline $\begin{array}{l}\text { Pregunta 6: ¿Conoces a qué hace referencia el } \\
\text { término acoso? En este sentido, ¿usted ha vivido } \\
\text { hechos de acoso en la universidad? ¿Por } \\
\text { quién/quienes? ¿Dónde? ¿Cómo se dio dicha } \\
\text { situación? }\end{array}$ & $\begin{array}{l}\text { “...Sí, sí como los comentarios, lo que te digo entonces } \\
\text { esos comentarios siempre reiterativos de que "eres } \\
\text { gay..." } \\
\text { "...Es que no lo haces, es que no sé qué” Pero qué estás } \\
\text { escondiendo ahí?..." } \\
\text { "Si, digamos que por mi parte, personalmente con los } \\
\text { maestros no me ha pasado... Bueno sí, o sea sí, ahora } \\
\text { que hago memoria sí, pero ahí hay una cosa diferente, } \\
\text { pero entonces si era como, el maestro está muy... todo el } \\
\text { tiempo está, estaba como haciéndome sentir mal pero, } \\
\text { pero como" "No es que tu no bailas, es que tu no sé } \\
\text { qué..." "Pero no era por eso, sino por otras cosas..." }\end{array}$ \\
\hline $\begin{array}{l}\text { Pregunta } 7: \text { ¿Conoces de alguien que ha sido víctima } \\
\text { de alguna forma de acoso en la universidad? ¿De } \\
\text { qué manera se dio el acoso? ¿Verbal, social, físico o } \\
\text { través de medios tecnológicos de comunicación? }\end{array}$ & $\begin{array}{l}\text { "...los hombres, pueden ser muy reiterativos y como muy } \\
\text { encima de la chica y, entonces, eso puede ser, pero no } \\
\text { sé hasta qué punto, pero si pasa, entonces que si la niña } \\
\text { esta linda, así como en todos los espacios que } \\
\text { "Ay que no sé qué" que el comentario "Que muy linda y } \\
\text { que no sé qué", pero claro llega también el punto en que } \\
\text { sí claro le dice de pronto, también se siente vulnerada..." }\end{array}$ \\
\hline
\end{tabular}

\section{DISCUSIÓN}

En seguida, se realiza la discusión de resultados del ACD empleando elementos de tipo cuantitativo y su relación con los sentimientos generados, tipos de violencias y posible origen de estas manifestaciones. En las categorias de 1) bullying, 2) acoso o abuso sexual y 3) discriminación, rechazo o exclusión social, se identificaron victimas y victimarios de los cuales es posible reconocer algunas expresiones del análisis de las narrativas.

$A C D$ en bullying desde el discurso de víctima. Este tipo de discurso fue identificado a partir de la posición que toma el sujeto frente al fenómeno y los sentimientos negativos que expresa resultado de estos sucesos. El análisis permitió visibilizar una situación política sobre el bullying, en la cual el sujeto busca las reivindicaciones emancipatorias y sus relaciones sociales a través de la descripción acerca de cómo el poder y la dominación se producen y reproducen en las prácticas sociales (Azambuja y Nogueira, 2009). Las unidades de contexto que se encontraron en este discurso fueron bullying, acoso/abuso sexual y rechazo/exclusión social. 
Tabla 4. Violencia de género: fragmento discursivo discriminación, rechazo o exclusión social.

\begin{tabular}{|c|c|}
\hline Pregunta & Fragmentos discursivos \\
\hline $\begin{array}{l}\text { Pregunta 8: ¿Crees que, por } \\
\text { tu género has sido } \\
\text { rechazado? ¿De qué } \\
\text { manera? ¿Dentro del } \\
\text { contexto universitario lo } \\
\text { evidenciaste? }\end{array}$ & 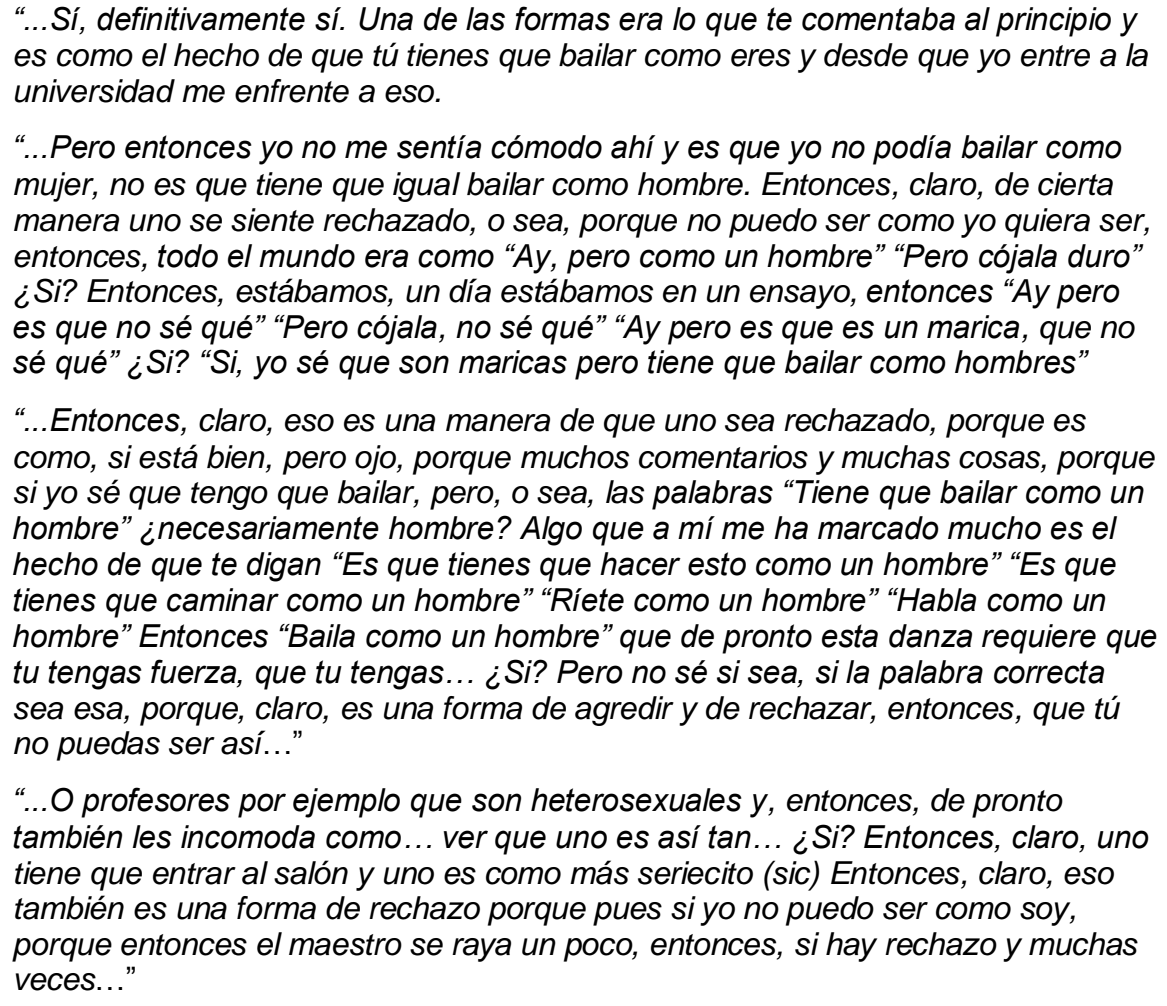 \\
\hline $\begin{array}{l}\text { Pregunta 9: ¿En qué } \\
\text { situaciones has } \\
\text { experimentado insultos, } \\
\text { rumores, amenazas, } \\
\text { comentarios mal } \\
\text { intencionados en la } \\
\text { universidad? ¿Has dejado } \\
\text { de participar en actividades } \\
\text { dentro de la universidad por } \\
\text { culpa de estos hechos? ¿Te } \\
\text { has sentido excluido de otra } \\
\text { manera en la universidad? }\end{array}$ & $\begin{array}{l}\text { "...Chévere que uno se dé un lugar y por como tú eres como persona y porque tú } \\
\text { eres como artista, más que porque seas gay o porque no seas gay. Porque, o sea, } \\
\text { a mí personalmente me pasa que, que siempre me puedo destacar en las danzas } \\
\text { y puedo hacer un papel de protagonista que es un hombre y lo puedo hacer } \\
\text { porque me he ganado ese espacio..." } \\
\text { "...el semestre pasado paso que éramos varios hombres...los hombres más } \\
\text { hombres y éramos nosotros, claro, yo me gane ese espacio, peor si yo hubiese } \\
\text { querido pues no, entonces yo también "Ay no, a usted no lo pongamos, porque } \\
\text { usted... toca es alguien que sea hombre" Pero pues uno mismo se va ganando } \\
\text { esos espacios y pues va diciendo como no, pues yo me levanto porque si yo } \\
\text { permito, claro, me desechan y entonces no "Ay mejor yo no soy protagonista" } \\
\text { Entonces, yo me he ganado esos espacios y he dicho no, yo quiero hacerlo } \\
\text { porque puedo hacerlo. O sea, porque yo puedo hacerlo, yo puedo hacer a este } \\
\text { personaje..." }\end{array}$ \\
\hline $\begin{array}{l}\text { Pregunta 10: ¿Alguna vez } \\
\text { has visto o has sido testigo } \\
\text { de hechos de acoso que } \\
\text { sobrepasen lo verbal en la } \\
\text { universidad? ¿Quiénes son } \\
\text { generalmente las personas } \\
\text { que sufren dichas } \\
\text { situaciones? }\end{array}$ & $\begin{array}{l}\text { “...Acoso, no... primero siento que cualquier persona puede sufrir de acoso, seas } \\
\text { hombre o seas mujer o seas niño o seas viejo o seas del género que seas. Siento } \\
\text { que cualquier persona es vulnerable, cualquier persona puede ser...Y eso es una } \\
\text { idea que tenemos que cambiar también tal vez, que es que el vulnerable eres tú, } \\
\text { es que los hombres también somos vulnerados, son los más, de pronto, violentos } \\
\text { y los más que vulneran. Sin embargo, hay poblaciones que son más vulnerables, } \\
\text { son más débiles de cierta manera, entonces, hace que sean vulnerables...” } \\
\text { "...son por ejemplo las mujeres...las ven débiles entonces, claro, es más fácil } \\
\text { aprovecharse de una mujer...” } \\
\text { "...las personas homosexuales, son vulneradas, porque entonces también no } \\
\text { sienten, de pronto también el apoyo de otros y entonces también se les ve como } \\
\text { ahí, abajo, chiquitos. Entonces, también son vulnerados, son muy vulnerados, o } \\
\text { sea la comunidad LGBTI es muy vulnerada, la mujer también es muy vulnerada...” }\end{array}$ \\
\hline
\end{tabular}

En el primer caso, se identificó en el discurso a los sujetos masculinos (64,3\%) como los principales protagonistas y ejecutores de los hechos de violencia, en escenarios productores de saboteos y críticas basadas en el género. Estos hallazgos son similares a los reportados por Chan et al. (2020) y Pörhölä et al. (2020), quienes indican que los hombres son los principales agresores de sus pares en espacios académicos universitarios, como se evidencian en Argentina, Estonia, Finlandia y Estados Unidos. 
Las agresiones más frecuentes que se identificaron fueron la imitación, burla, morbo, chiste, bromas e irrespeto en el contexto académico del programa y universitario, y que en un 35,7\% las víctimas fueron homosexuales o lesbianas En consecuencia, se infieren sentimientos negativos, por ejemplo, expresiones críticas con respecto a la condición machista existente y su aceptación en los espacios de formación. En este sentido, aquellos actos de violencia están siendo socialmente institucionalizados. Por consiguiente, se logra establecer que dicho discurso tiene incidencia en los seres humanos en el sentido de que su performatividad construida (es un hacer constante y permanente) desde la diferencia es agredida y motivo de mofa social, condicionando sus actos y libre expresión en los espacios académicos, lo cual es confirmatorio de la matriz cultural hegemónica masculina de construcción de identidad (Butler, 2017). Finalmente, se pone de manifiesto la transmutación de este fenómeno según el discurso de un estudiante universitario, ya que ello se constituye como un problema de salud pública y derechos humanos vulnerados, situación que requiere procesos de intervención social inmediata (Azambuja y Nogueira, 2009).

$A C D$ en acoso o abuso sexual desde el discurso de víctima. En lo que respecta a esta categoría, se encontró que de los sujetos mencionados los docentes y maestros de género masculino $(71,4 \%)$ fomentan prácticas patriarcales sobre sus estudiantes y que las mujeres $(28,6 \%)$ son las principales perjudicadas de tales acciones, esto también fue hallado en un estudio sobre el acoso en contra de las mujeres, donde estos hechos se manifiestan porque son un fenómeno complejo y multidimensional, que tiene un carácter polifacético que implican cuestiones subjetivas y culturales sobre el género (Rocha Aguiar et al, 2020). Esto representa una forma de subordinación y orden social que son reproducidos por la diferencia de género manifestado en prácticas sociales violentas, con un lenguaje sexista y excluyente. El tipo de agresión fue acoso en actividades académicas en las asignaturas propias del programa de artes escénicas. Los sentimientos hallados durante el análisis fueron negativos, pues se encontraron en la entrevista expresiones como: "estaba como haciéndome sentir mal", "el maestro está muy... todo el tiempo está... haciéndome sentir mal...", "No, es que tu no bailas, es que tu no sé qué... Pero no era por eso, sino por otras cosas..." y "entonces, esos comentarios siempre reiterativos de que eres gay...". Esto enuncia un contexto excluyente de homofobia que puede ser causado desde el victimario por su rechazo social a la diversidad de género, su autodefinición de género, la orientación sexual con la cual se identifica, la religión que profesa y el número de parejas sexuales que ha tenido (Moral y Valle, 2014).

$A C D$ en discriminación, rechazo o exclusión social desde el discurso de víctima. Los sujetos que ejercen este fenómeno son de género masculino (100\%) y en su discurso se logra identificar como macho, hombre y maestro. Dichos sujetos organizan y realizan de forma sistemática la implementación de los rasgos patriarcales como insultos, comparaciones, rechazo, frustración, vulnerabilidad, marginación y humillación en el ambiente académico del programa. Los sentimientos generados son negativos pues incurre en acciones basadas en violencia psicológica en las que hay una relación unilateral y estructurada alrededor de un agresor y una víctima (Parrini, 2013). Es el caso de la respuesta en las preguntas 8 y 10 (Tabla 4), en ella encontramos una respuesta afirmativa si desde su punto de vista cree que ha sido rechazado, y para delimitar las situaciones encontramos las siguientes expresiones: "de cierta manera uno se siente rechazado... porque no puedo ser como yo quiera ser" "sí, yo sé que tengo que bailar, pero... tiene que bailar como un hombre ¿necesariamente hombre?..." "Algo que a mí me ha marcado mucho es el hecho de que te digan es que tienes que hacer esto como un hombre, es que tienes que caminar como un hombre, ríete como un hombre, habla como un hombre, entonces baila como un hombre". En concordancia con esto, se han encontrado un estudio de Vanyoro (2020) donde analiza que el lenguaje en espacios universitarios es sistémico y estructurado que normaliza la opresión, lo que genera relaciones de poder, privilegio, marginalidad en términos de género y sexualidad que tienen un origen en los sistemas políticos que se establecieron que dieron origen a la instituciones de educación superior.

$A C D$ en bullying desde el discurso de victimario. Los victimarios son mayoritariamente hombres (66,7\%) y las víctimas pertenecen al género femenino o masculino y se identifican como femenino (33,3\%). Estos hallazgos se relacionan con el estudio de Musharraf et al. (2020), el cual presenta una alta participación de hombres en el acoso tradicional que tiene como víctima a las mujeres. Este discurso se observó en las afirmaciones positivas de actos que involucran hechos de violencia, es decir, el entrevistado admite que violenta en ocasiones a otros de forma similar a como lo agreden a él, cuando es ubicado en el discurso de víctima "uno busca chistes y bromas, pero uno las encuentra más fácil en esas personas", refiriéndose a aquellos que pertenecen a la diversidad de género, en particular a los homosexuales. El estudio de Wang et al. (2020) confirma lo anterior, las personas que son intimidadas, también intimidan a otros, por ello, sugieren como estrategia de mitigación el afrontamiento positivo con grupos de víctimas y victimarios en el mismo espacio. Los sentimientos en este caso son neutros, puesto que no menciona aprobación, refutación o consecuencia en sus acciones, en otras palabras, no tiene una posición desfavorable de sus actos. El tipo de agresión corresponde a la imitación, en concreto, chistes y bromas basados en la violencia de género vivida en las aulas de la institución. 
$A C D$ en acoso o abuso sexual desde el discurso de testigo directo. Los sujetos que efectúan según el discurso de testigo directo prácticas de acoso o abuso sexual (victimarios) son docentes de género masculino y compañeros de estudio (60\%), hacia las mujeres (víctimas) (40\%). Estos hallazgos se relacionan en parte con los resultados de Raboteg-Šarić y Bartaković (2019) y Davis et al. (2018), quienes observaron que los niños y estudiantes que se identifican como no heterosexuales cumplen roles de matoneo y acosador, más que las niñas y mujeres. Afecto y afinidad con la población académica que lo rodea, son los sentimientos hallados, valga como ejemplo lo dicho por el entrevistado: "hay cierta complicidad y hay cierto afecto entre todos, porque somos artistas, porque compartimos un espacio". Él se refiere a sí mismo como observador, respecto al tipo de agresión, la relación de los otros frente a los hechos y la posición se considera favorable, en cuanto a que no se registra oposición. Ello presenta similitudes con el estudio de Chan et al. (2020), con respecto a la teoría de atribución defensiva, puesto que el estudiante presenta respuestas basadas en la empatía con los victimarios de acuerdo con el contexto. De igual manera, se precisan prejuicios de comportamiento delante de hombres y mujeres.

\section{Análisis de estructura}

Esta sección del ACD se enfoca en identificar los fragmentos e hilos discursivos presentes en la unidad de contexto con el propósito de reconocer los elementos del discurso a través de los actos de habla, como lo plantea Merino (1998) con base en los marcadores lingüísticos establecidos por Vand Dijk, en su taxonomía. Después, se desarrolla el enfoque cualitativo para determinar la finalidad, funcionalidad y tipo de acto de habla en cual se halló el discurso. En la tabla 5 se encuentra el consolidado de los resultados para las unidades de contexto estudiadas.

Tabla 5: Resultados análisis cualitativo del discurso.

\begin{tabular}{|l|l|l|l|}
\hline $\begin{array}{c}\text { Unidad de } \\
\text { contexto }\end{array}$ & \multicolumn{1}{|c|}{ Bullying } & \multicolumn{1}{|c|}{ Acoso, abuso sexual } & Rechazo, exclusión social \\
\hline $\begin{array}{l}\text { Finalidad del } \\
\text { discurso }\end{array}$ & $\begin{array}{l}\text { Dar a conocer el tipo de manifestaciones de } \\
\text { violencia de género en ámbitos universitarios } \\
\text { de contextos sociales y académicos }\end{array}$ & $\begin{array}{l}\text { Dar a conocer la } \\
\text { comunicación que hay en } \\
\text { los estudiantes y docentes } \\
\text { universitarios. }\end{array}$ & $\begin{array}{l}\text { Dar conocer las formas de } \\
\text { rechazo y exclusión durante } \\
\text { la formación docente en } \\
\text { artes escénica. }\end{array}$ \\
\hline Emisores & $\begin{array}{l}\text { Los profesores, personal de apoyo } \\
\text { colaborativo a las instituciones y compañeros } \\
\text { de estudio sin precisar el género. }\end{array}$ & Hombres & Profesores \\
\hline Receptores & Homosexuales y bisexuales. & Mujeres & $\begin{array}{l}\text { Homosexuales, mujeres y } \\
\text { hombres }\end{array}$ \\
\hline $\begin{array}{l}\text { Tipo de } \\
\text { discurso }\end{array}$ & Expresivo & Representativo & Representativo \\
\cline { 3 - 4 } & & & Conmisivo \\
\cline { 2 - 4 }
\end{tabular}

La primera unidad de contexto analizada es el bullying. La finalidad del discurso es dar a conocer el tipo de manifestaciones de violencia de género en ámbitos universitarios de contextos sociales y académicos, desde su sinceridad, los emisores son los profesores, personal de apoyo colaborativo a las instituciones y compañeros de estudio sin precisar el género; los homosexuales y bisexuales, son los receptores de dicha violencia, las prácticas más comunes fueron burlas, chistes y comentarios malintencionados o inadecuados hechos por los emisores en ambientes académicos. Los tiempos verbales del relato son el indicativo presente y el indicativo condicional, uno refiere a situaciones concretas de actos de agresión y el otro, a la acción reflexiva de los emisores en torno a sus comportamiento en relación con las personas que pertenecen a la diversidad de género. Estas prácticas se llevan a cabo en ambientes sociales, como por ejemplo, cafeterías. Por último, el tipo de discurso es expresivo, para ilustrar mejor, afirmaciones repetitivas con el sentir frente a lo que sucede, como fue el caso de "siento que no es como tal en un lugar específico, porque siento que todos los lugares pueden ser esos espacios y por ejemplo pasa acá en la cafetería".

La segunda unidad de contexto estudiada es el acoso o abuso sexual. La finalidad del discurso es dar a conocer la comunicación que hay entre estudiantes y docentes-estudiantes. En el primer caso, se definió como fraterna (ambos emisores y receptores), a saber: "somos muy cariñosos..." "...somos como muy amigos..." "...si hay mucha forma de afecto" “...siempre hay besos y abrazos...". En el segundo caso, esa comunicación evidencia enfrentamiento, que además, es en una dirección emisor (docente) a receptor (estudiante), otro ejemplo ilustrativo fue: "...entonces, esos comentarios siempre reiterativos de que eres gay" "Es que no lo haces, es que no sé qué" "es una piedra en el zapato pero es por otra cosa...". No obstante, frente a los hechos de acoso relaciona a los hombres de forma general sobre las mujeres con actos de persecución y comentarios mal intencionados: "los hombres, pueden ser muy reiterativos y como muy encima 
de la chica y entonces eso puede ser... entonces que si la niña esta linda, así como en todos los espacios... el comentario "Que muy linda..." pero claro llega también el punto en que...también se siente vulnerada". El tiempo verbal del discurso es indicativo presente y se infiere que las aulas de clase son el lugar donde este se lleva a cabo. El tipo de discurso es representativo, puesto que es la representación de la verdad por parte del hablante sobre su experiencia al mundo.

La tercera unidad de contexto examinada es el rechazo o exclusión social. La finalidad del discurso es dar a conocer las formas de rechazo y exclusión en la cual se ha visto involucrado durante su proceso de formación docente en artes escénicas, especialmente en asignaturas relacionadas con la expresión corporal. Los emisores son profesores de las áreas mencionadas anteriormente, mientras que las mujeres, hombres y homosexuales son los receptores, lo cual en las siguientes expresiones se evidencia: "las ven débiles, entonces, claro, es más fácil aprovecharse de una mujer", "son los más...violentos y los más que vulneran" y “...la comunidad LGBTI es muy vulnerada”. Los actos de rechazo y exclusión son frecuentes en las actividades académicas, por ejemplo, en las clases de danza se registran frases de este tipo, así: “...yo no podía bailar como mujer, no, es que tiene que igual bailar como hombre" "Ay, pero es que es un marica... yo sé que son maricas, pero tienen que bailar como hombres..." "...tiene que bailar como un hombre..." "...es que tienes que caminar como un hombre" "...ríete como un hombre..." "...habla como un hombre..." "...tiene que bailar como un hombre". El tiempo verbal del discurso es indicativo presente y ocurre en el espacio de formación académica de artistas. El tipo de manuscrito que predomina es representativo y en menor medida expresivo y conmisivo.

\section{DISCUSIÓN FINAL}

Las relaciones de poder presentes en el discurso de género, en particular en un estudiante de educación superior que pertenece al programa de licenciatura en artes escénicas, son desiguales. Los sistemas de socialización universitaria determinan y reproducen en gran medida los mecanismos de violencia de género sobre la diversidad de género y las mujeres. Dichos mecanismos corresponden a ideologías machistas y patriarcales que desconocen y rechazan la diversidad de género. La violencia de género hallada en el discurso es psíquica y social, la primera tiene como característica principal que el victimario es el hombre. La mujer y la diversidad de género son sus víctimas, los actos más frecuentes son la presión psicológica sobre temas académicos, remedo, frustración, chistes y bromas de mal gusto. La segunda ubica en la relación de poder al hombre, maestro y profesor como victimario y a la mujer en el rol de víctima, los hechos más recurrentes son sabotaje, descalificación y estereotipos. La tercera y última, contiene a la mujer en el papel de víctima y al hombre, docente, profesor, compañeros hombres y machos en el de victimario, las prácticas más comunes son el sabotaje, la descalificación y la presión psicológica en relación con el proceso académico.

Sin embargo, lo anterior no es estático. El estudiante se ubica discursivamente (víctima, victimario y testigo) con base en el sentimiento y lugar social que ocupa en un momento y espacio determinado, es decir, sus sentimientos son negativos cuando es víctima en áreas comunes y de socialización (cafeterías y pasillos), neutros en el rol de victimario en el aula de clases y afinidad como testigo directo en diferentes escenarios de formación académica. Todo lo anterior conduce a repensar el proceso de formación docente a través del acto comunicativo para el reconocimiento del otro, valga decir, la creación al interior del programa de artes escénicas, de estrategias de intervención cultural y social que fortalezcan y desarrollen conocimientos en género, otredad y elimine la violencia de género.

\section{CONCLUSIONES}

De acuerdo al trabajo presentado, a la discusión desarrollada y a los resultados obtenidos, se pueden plantear las siguientes conclusiones principales:

1.- El uso del ACD es importante para la comprensión de los discursos de género inmersos en las narrativas de los actores educativos, debido a que permite conocer los sentidos y significados de las relaciones entre pares y con los docentes en la academia, o sea, esto contribuye a la discusión de resultados con estudios de muestras representativas.

2.-En las categorias de bullying, acoso o abuso sexual y discriminación, rechazo o exclusión social se encontró que existen prácticas que manifestan violencia de género en el contexto de educación superior ubicado en el sur de Bogotá, lo cual genera condicionamiento de participación de actividades académica; los principales agresores son hombres y la victimas son las mujeres o las personas que pertenecen a la diversidad de género. Se halló principalmente relaciones de poder que oprimen en aptitudes y vulnera psicológicamente. El uso de un lenguaje sexista en el ámbito académico podría originarse por múltiples factores, entre ellos los de carácter social de los sujetos que hacen parte del contexto o de carácter histórico en la cual se creó la institución de educación superior. 
3.- La violencia de género que presenta el discurso del estudiante universitario se relaciona con la reproducción de la matriz cultural hetero masculina en las instituciones educativas, la cual esta mediada por los roles víctima, victimario y testigo, además, el estudiante asume estos de acuerdo con el grupo social y contexto donde se desarrolla la agresión, es decir, el rol se caracteriza por ser cambiante.

\section{REFERENCIAS}

Ahmad, M. y Shah, S.K., A Critical Discourse Analysis of Gender Representations in the Content of 5th Grade English Language Textbook, 10.17583/rimcis.2019.3989, RIMCIS-International and Multidisciplinary Journal of Social Sciences, 8(1), 1-24 (2019)

Álvarez-Aguilar, N.T., González-Duéñez, V.P., y Castillo-Elizondo, J.A., Mujeres y carreras de ingeniería en la Universidad Autónoma de Nuevo León, en México: una Mirada desde las Vivencias de las Estudiantes, https://doi.org/10.4067/S0718-50062019000400085, Formación Universitaria, 12(4), 85-94 (2019)

Azambuja, M.P., y Nogueira, C., Potencialidades investigativas para a violência de gênero: utilização da análise de discurso, https://doi.org/10.1590/s1413-81232009000500013, Ciência \& Saúde Coletiva, 14(5), 1721-1730 (2009)

Ballester, I., El cuerpo abierto. Representaciones extremas de la mujer en el arte contemporáneo, 1-516, Trea, Gijón, España (2012)

Butler, J., El género en disputa, 1aㅡ. Ed., Bogotá, Colombia (2017)

Chan, H.C., Sheridan, L., y Adjorlolo, S., Stalking and intrusive behaviors in ghana: Perceptions and victimization experiences, 10.3390/ijerph17072298, International Journal of Environmental Research and Public Health, 17(7), 1-19 (2020)

Davis, E., Campbell, M., y Whiteford, C., Bullying victimization in non-heterosexual university students, 10.1080/10538720.2018.1463887, Journal of Gay and Lesbian Social Services, 30(3), 299-313 (2018)

Devís-Devís, J., Pereira-García, S. y otros 3 autores, Looking back into trans persons' experiences in heteronormative secondary physical education contexts, https://doi.org/10.1080/17408989.2017.1341477, Physical Education and Sport Pedagogy, 23(1), 103-116 (2018)

Dalton, E., A feminist critical discourse analysis of sexual harassment in the Japanese political and media worlds, 10.1016/j.wsif.2019.102276, Women's Studies International Forum, 77, 102276, 1-9 (2019)

Fernández, F., El análisis de contenido como ayuda metodológica para la investigación, Ciencias Sociales, 96, 35-53 (2002)

García-Díaz, V., Fernández-Feito, A., y otros 3 autores, Tolerance of intimate partner violence and sexist attitudes among health sciences students from three spanish universities, 10.1016/j.gaceta.2019.01.003, Gac Sanit., 34(2), 179185 (2020)

Kelmendi, K., y Baumgartner, F., Exploring violence socialization and approval of intimate partner violence among university students in Kosovo, 10.1177/0886260517692336, Journal of Interpersonal Violence, 35(5-6), 1081-1107 (2020)

Lewis, R., y Anitha, S., Explorations on the Nature of Resistance: Challenging Gender-Based Violence in the Academy, Palgrave Studies in Gender and Education, Palgrave, 75-94, Maroochydore, Australia, (2019)

Merino, E., Estudio comparativo de actos de habla en dos tipos de narrativa oral Mapuche, https://doi.org/10.7764/onomazein.47.10, Onomázein: Revista de lingüística, filología y traducción, 3, 163-176 (1998)

Moral, J., y Valle, A., Predictive models of attitude toward homosexuality in heterosexual men, http://dx.doi.org/10.11144/Javeriana, Universitas Psychologica, 13(4), 1395-1407 (2014)

Musharraf, S., Bauman, S., Anis-ul-Haque, M., y Malik, J.A., General and ICT self efficacy in different participants roles in cyberbullying/victimization among Pakistani university students, 10.3389/fpsyg.2019.01098, Front. Psychol., 10, 1098, 1-11 (2019)

Osuna-Rodríguez, M., Rodríguez-Osuna, L.M., Dios, I., y Amor, M.I., Perception of gender-based violence and sexual harassment in university students: Analysis of the information sources and risk within a relationship, 10.3390/ijerph17113754, Int. J. Environ. Res. Public Health, 17(11), 1-14 (2020)

Pörhölä, M., Cvancara, K., y otros 4 autores, Bullying in university between peers and by personnel: Cultural variation in prevalence, forms, and gender differences in four countries, 10.1007/s11218-019-09523-4, Social Psychology of Education, 23(1), 143-169 (2020)

Raboteg-Šarić, Z., y Bartaković, S., Empathy and moral disengagement as predictors of bystander roles in school bullying, 10.5457/p2005-114.248, Central European Journal of Paediatrics, 15(2), 161-176 (2019)

Ramos-Jiménez, A., Hernández-Torres, R.P., Murguía-Romero, M., y Villalobos-Molina, R., Prevalence of bullying by gender and education in a city with high violence and migration in Mexico, Rev. Panam. Salud Publica, 41(12), 1-6 (2017) 
Rocha Aguiar, F.A., Magalhães da Silva, R., y otros 4 autores, Vocational training and sexual assault against women: challenges for graduation in nursing, https://doi.org/10.1590/2177-9465-ean-2019-0135, Esc. Anna Nery, 24(1), 1-10 (2020)

Ruiz, A.E., Evangelista, A.A., y Xolocotzi, A., ¿Cómo llamarle a lo que tiene muchos nombres? ¿Bullying, violencia de género, homofobia o discriminación contra personas LGBTI?, http://dx.doi.org/10.24201/eg.v4i0.210 Revista Interdisciplinaria de Estudios de Género de El Colegio de México, 4, 1-31 (2018)

San Segundo, R., y Codina-Cadet, A., Enunciación de la violencia de género y marco educativo para su prevención, http://dx.doi.org/10.17583/generos.2019.4000, Multidisciplinary Journal of Gender Studies, 8(1), 26-47 (2019)

SIVIGE, Sistema Integrado de Violencias de Género, Colombia, http://onviolenciasgenero.minsalud.gov.co/ (2018)

Tekbas, S., y Pola, G., Evaluation of gender equality attitudes of turkish and foreign nursing students at a private university, 10.12669/pjms.36.5.2365, Pakistan Journal of Medical Sciences, 36(5), 884-889 (2020)

Van Dijk, T.A., El discurso como interpretación en la sociedad, El discurso como interacción social, 1aㅡ., vol 2,19-66, Barcelona, España (2000)

Vandana, Dalit girls and sexual harassment in the university, 10.1177/0971521519891478, Indian Journal of Gender Studies, 27(1), 33-54 (2020)

Vanyoro, K., Learning how language is used in higher education to strategically marginalise female, queer, and gender non-conforming people: an autoethnographic account, https://dx.doi.org/10.17159/2221-4070/2020/v9i0a1, Educ. res. soc. change, 9(spe), 1-14 (2020)

Wang, Y., Yu, H., y otros 6 autores, The victim-bully cycle of sexual minority school adolescents in China: prevalence and the association of mood problems and coping strategies, 10.1017/S2045796020000918, Epidemiology and Psychiatric Sciences, 29, e179, 1-12 (2020)

Woka, R.M., Métodos de análisis crítico del discurso. 1ª Ed., Gedisa, España (2003) 